\title{
Anti-Locality of the One-half Power of Elliptic Differential Operators
}

By

\author{
Kyûya MAsudA*
}

In connection with the question concerning the ring of operators generated by quantized field operators with a given region of space, using the edge of the wedge theorem, H. Reeh and S. Schlieder [3] showed that the one-half power $\left(m^{2} I-\Delta\right)^{1 / 2}$ of $m^{2} I-\Delta\left(\right.$ on $\left.R^{N}\right)$ has the anti-local property in the sense that if $f$ and $\left(m^{2} I-\Delta\right)^{1 / 2} f\left(f \in \mathbf{L}^{2}\left(R^{N}\right)\right)$ vanish in some nonempty open set $U$ in $R^{N}$, then $f(x)$ must be identically zero in $R^{N}$. I. Segal and R. Goodman [4] generalized the result of Reeh and Schlieder, and showed that $\left(m^{2} I-\Delta\right)^{\lambda}(\lambda$ : non-integral number) is also anti-local if the space dimension $N$ is odd. Recently, M. Murata [2] succeeded, by the so-called method of decent, in excluding the assumption that the space dimension is odd. The purpose of the present paper is to give another proof for the theorem of Reeh and Schlieder on the antilocality of $\left(m^{2} I-\Delta\right)^{1 / 2}$. The proof may be of some interest in two respects; it is simple, and secondly the method is applicable to the case of the one-half power of elliptic differential operators with variable coefficients, especially $(V(x)-\Delta)^{1 / 2}(V(x)$ : external potential).

Now let $\Omega$ be a domain with smooth boundary in an $N$-dimensional Euclidean space $R^{N}$. We define the operator $A$ in $\mathbf{L}^{2}(\Omega)$ by:

$$
\begin{aligned}
& \mathbf{D}(A)=\left\{u \in \mathbf{H}^{2}(\Omega) ; u=0 \text { on the boundary of } \Omega\right\} \\
& A u=\sum_{j, k=1}^{N} \frac{\partial}{\partial x_{j}}\left(a_{j k}(x) \frac{\partial u}{\partial x_{k}}\right)+a(x) u, \quad u \in D(A),
\end{aligned}
$$

Received March 7, 1972.

Communicated by H. Araki.

* Department of Mathematics, University of Tokyo, Tokyo 113, Japan. 
where $\mathbf{H}^{2}(\Omega)$ is the set of all $\mathbf{L}^{2}(\Omega)$-functions whose distribution derivatives up to order 2 are in $\mathbf{L}^{2}(\Omega)$. We assume:

(i) $a_{j k}(x)(j, k=1, \ldots, N)$ are bounded and continuously differentiable real valued functions on $\bar{\Omega}$ (the closure of $\Omega$ ) and $a(x)$ is bounded and continuous on $\bar{\Omega}$.

(ii) $\sum_{j, k=1}^{N} a_{j k}(x) \zeta_{j} \zeta_{k} \geqq \delta|\zeta|^{2}$ ( $\delta$ : some positive constant) for all $x \in \Omega$ and $\zeta \in R^{N}$.

As is well known, the operator $A$ is a self-adjoint operator bounded from above in $\mathbb{L}^{2}(\Omega)$. Our theorem is as follows:

Theorem. The one-half power $(-A)^{1 / 2}$ of $-A$, has the anti-local property: if $f$ and $(-A)^{1 / 2} f\left(f \in \mathbb{D}\left(A^{1 / 2}\right)\right)$ vanish in some nonempty open set $U$ in $\Omega$, then $f$ must be zero almost everywhere in $\Omega$, where $(-A)^{1 / 2}$ is defined through the spectral representation of $A$.

As a direct consequence of the above theorem, we can see that $\left(m^{2} I-\Delta\right)^{1 / 2}$ is anti-local in $\mathbb{L}^{2}\left(R^{N}\right)$.

Proof. Since the operator $-A$ is a self-adjoint operator bounded from below in $\mathbf{L}^{2}(\Omega)$, we see that

$$
U(t)=\exp \left(i t(-A)^{1 / 2}\right), \quad-\infty<t<\infty,
$$

defines the one-parameter family of group of bounded operators in $\mathbb{L}^{2}(\Omega)$. If we set

$$
u(x, t)=(U(t) f)(x)
$$

where $f(x)$ is the function given in the theorem, then $u(x, t)$ is a (generalized) solution of the wave equation

$$
\frac{\partial^{2} u}{\partial t^{2}}=A u
$$

i.e.,

$$
\frac{\partial^{2} u}{\partial t^{2}}=\sum_{j, k=1}^{N} \frac{\partial}{\partial x_{j}}\left(a_{j k}(x) \frac{\partial u}{\partial x_{k}}\right)+a(x) u
$$


with the initial condition

$$
u(x, 0)=f(x), \quad \frac{\partial}{\partial t} u(x, 0)=i\left((-A)^{1 / 2} f\right)(x)
$$

Since a solution of the wave equation has the finite propagation speed of disturbance, and since $u(x, 0)=\frac{\partial}{\partial t} u(x, 0)=0$ in $U$ by the assumption, there exist a $t_{0}>0$ and a nonempty open subset $U_{0}$ of $U$ such that

$$
u(x, t)=0
$$

for $0<t<t_{0}$ and $x \in U_{0}$.

Hence, if $\varphi$ is a $C^{\infty}$-function with compact support in $U_{0}$ i.e., $\varphi \in C_{0}^{\infty}\left(U_{0}\right)$, then we have

$$
(u(\cdot, t), \varphi)=0
$$

for $0<t<t_{0}$, where $(\cdot, \cdot)$ is the inner product of $\mathbb{L}^{2}(\Omega)$. Hence the function $F(z)$ defined by

$$
F(z)=\left(\exp \left(i z(-A)^{1 / 2}\right) f, \varphi\right), \quad \operatorname{Im} z \geqq 0,
$$

has the following properties:

(i) $F(z)$ is holomorphic in $\operatorname{Im} z>0$, and is continuous in $\operatorname{Im} z \geqq 0$

(ii) $F(t)=(u(\cdot, t), \varphi) \quad$ (for real $t$ )

(iii) $F(t)=0 \quad$ (for $0<t<t_{0}$ )

By the Schwarz reflection principle in the complex function theory, $F(z)$ can be continued holomorphically across the interval $\left(0, t_{0}\right)$ into the lower half-plane $\operatorname{Im} z<0$. Hence we see from (iii) that

$$
F(z)=0, \quad \operatorname{Im} z \geqq 0 .
$$

In particular, we have

$$
F(t)=(u(\cdot, t), \varphi)=0, \quad-\infty<t<\infty .
$$

Since $\varphi$ is an arbitrary function in $\mathbf{C}_{0}^{\infty}\left(U_{0}\right)$, we have 


$$
u(x, t)=0
$$

in the cylindrical domain $\left\{(x, t) ; x \in U_{0},-\infty<t<\infty\right\}$.

By the unique continuation theorem for solutions of the wave equation (K. Masuda [1]), we have, for any $t$,

$$
u(x, t)=0, \quad \text { a.e. } x \text { in } \Omega .
$$

In particular, $u(x, 0)=f(x)=0$, a.e. $x$ in $\Omega$.

This proves the theorem.

\section{References}

[1] Masuda, K., A unique continuation theorem for solutions of wave equations with variable coefficients, J. Math. Anal. Appl. 21 (1968), 369-376.

[2] Murata, M., Anti-locality of certain functions of the Laplace operator, to appear.

[3] Reeh, H. and S. Schlieder, Bemerkungen zur Unitäräquivalenz von Lorentzinvarianten Feldern, Nuovo Cimento, 22 (1961), 1051-1068.

[4] Segal, I. and R. Goodman, Anti-locality of certain Lorentzinvariant operators, J. Math. Mech. 14 (1965), 629-638. 